\title{
Chinese expert consensus on coronavirus disease 2019 vaccination for breast cancer patients
}

\author{
Jianyun $\mathrm{Nie}^{1 \#}$, Tao Wang ${ }^{2 \#}$, Ruonan $\mathrm{Xu}^{3 \#}$, Jianbin $\mathrm{Li}^{2}$, Yongmei Yin ${ }^{4}$, Xiaojia Wang ${ }^{5}$, Cuizhi Geng ${ }^{6}$, \\ Fu-Sheng Wang ${ }^{3}$, Erwei Song ${ }^{7}$, Zefei Jiang ${ }^{2}$ \\ ${ }^{1}$ Department of Breast Cancer, Yunnan Cancer Center, Third Affiliated Hospital of Kunming Medical University, Kunming, China; ${ }^{2}$ Senior \\ Department of Oncology, the Fifth Medical Center of PLA General Hospital, Beijing, China; ${ }^{3}$ Department of Infectious Disease, the Fifth Medical \\ Center of PLA General Hospital, Beijing, China; ${ }^{4}$ Department of Breast Center, the First affiliation Hospital with Nanjing Medical University, \\ Nanjing, China; ${ }^{5}$ Department of Chemotherapy, Cancer Hospital Affiliated to Chinese Academy of Sciences, Hangzhou, China; ${ }^{6}$ Department of \\ Breast Center, Fourth Hospital Affiliated to Hebei Medical University, Shijiazhuang, China; ${ }^{7}$ Breast Tumor Center, Sun Yat-sen Memorial Hospital, \\ Sun Yat-sen University, Guangzhou, China \\ \#These authors contributed equally to this work. \\ Correspondence to: Fu-Sheng Wang. Department of Infectious Disease, the Fifth Medical Center of PLA General Hospital, Beijing, China. \\ Email: fswang302@163.com; Erwei Song. Breast Tumor Center, Sun Yat-sen Memorial Hospital, Sun Yat-sen University, Guangzhou, China. Email: \\ songerwei02@aliyun.com; Zefei Jiang. Senior Department of Oncology, the Fifth Medical Center of PLA General Hospital, Beijing, China. Email: \\ jiangzefei@csco.org.cn.
}

\begin{abstract}
Coronavirus disease 2019 (COVID-19) vaccination is one of the most important interventions for establishing herd immunity, reducing the rate of infections and deaths, and achieving effective prevention and control of the pandemic. Furthermore, vaccination for vulnerable populations, including the elderly, children, and tumor patients, has become a key issue during the fight against this pandemic. However, most vaccine clinical trials did not include patients with advanced malignancies; moreover, there are no available data on the safety and efficacy of COVID-19 vaccines for this population. Although the viral vectors for adenoviral vector vaccines used are replication deficient, there are no existing safety data on the use of the same vaccine types. Therefore, cancer patients should be fully informed about the potential benefits and risks of COVID-19 vaccination. During the COVID-19 pandemic, the diagnosis and treatment patterns of breast cancer have changed according to the specific grade and stage of the malignancy to ensure the safety of patients. However, there is no direct evidence of whether breast cancer patients can receive COVID-19 vaccination and the timing of vaccination. To promote the rational COVID-19 vaccination in breast cancer patients, the Chinese Society of Clinical Oncology-Breast Cancer held an online expert meeting on September 9, 2021, during which participants voted on issues related to vaccination for breast cancer patients in China and created expert recommendations on different breast cancer grades to guide clinical practice.
\end{abstract}

Keywords: Breast cancer; COVID-19 vaccination; Chinese consensus; CSCO BC

Received: 11 September 2021; Accepted: 28 September 2021; Published: 31 October 2021.

doi: $10.21037 /$ tbcr-21-31

View this article at: https://dx.doi.org/10.21037/tbcr-21-31

\section{Introduction}

As of September 2021, more than 220 million confirmed coronavirus disease 2019 (COVID-19) cases and 4.7 million deaths have been reported worldwide (1). Vaccination is one of the most important interventions for establishing herd immunity, reducing the rate of infections and deaths, and achieving effective prevention and control of the pandemic.

The Chinese government has made significant efforts and contributions to prevent and control the COVID-19 pandemic. Aside from maximizing the unconditional and active treatments for COVID-19 patients in China, the 
Table 1 Comparison of the currently available COVID-19 vaccines

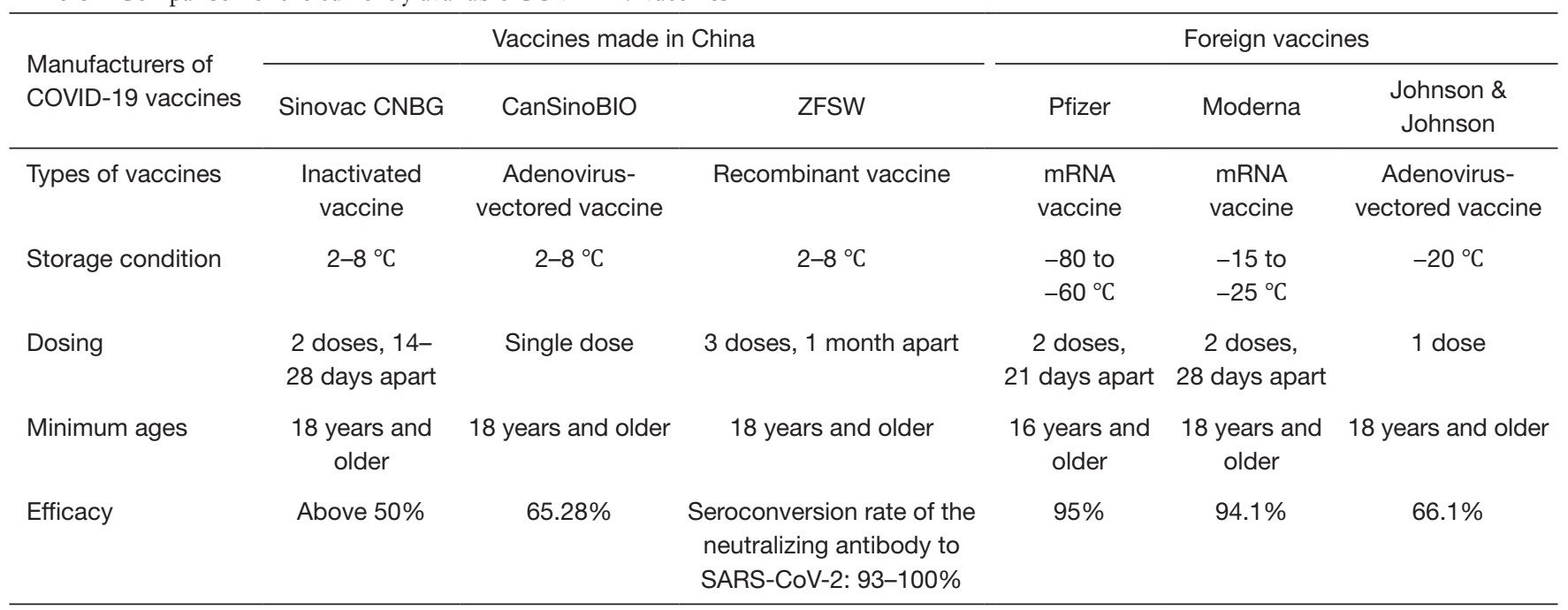

Chinese government has made every effort to promote universal COVID-19 vaccination by conducting awarenessraising activities and developing a vaccination program to achieve herd immunity (2). According to the National Health Commission of China, more than 2.1 billion doses of COVID-19 vaccines were administered across China as of September 13, 2021 (3). Therefore, vaccination for vulnerable populations, including older adults, children, and patients with tumors, has become a key issue during the fight against COVID-19.

According to the latest estimates on the global burden of cancer released by the International Agency for Research on Cancer, the number of new cases has increased to 19.29 million in 2020. Notably, there were 2.26 million new breast cancer cases, surpassing lung cancer as the most commonly diagnosed cancer worldwide (4). Patients with cancer are at a high risk of developing severe or critical COVID-19 due to their weakened/disordered immune function and frequent treatment-seeking behaviors (5). Recent data indicate that cancer patients account for more than $2 \%$ of all COVID-19 patients, and have a significantly higher incidence of serious adverse events (including admission to the intensive care unit, invasive ventilation, or death) than non-cancer patients (6). Therefore, sufficient immune protection for cancer patients is highly valuable for lowering the mortality rate among COVID-19 patients.

However, most vaccine clinical trials did not include patients with advanced malignancies; moreover, there are no available data on the safety and efficacy of COVID-19 vaccines for this population. Most of the clinical evidence on COVID-19 vaccination in patients with cancer were based on the results of influenza vaccination research. Considering the safety and efficacy of influenza vaccination in the populations with tumors, many academic groups in China and abroad recommended that cancer patients should be prioritized for COVID-19 vaccination to reduce the risks of infection and severe illness in this population considering the global shortage of COVID-19 vaccines (7).

However, COVID-19 prevention and control dramatically differ in China and abroad, and there are diverse recommendations on the necessity and timing of COVID-19 vaccination and the specific vaccine(s) for cancer patients and other special populations (Table 1). According to the Tecbnical Guide to COVID-19 Vaccination (first edition) (8) jointly issued by the National Health Commission and the State Administration of Traditional Chinese Medicine of the People's Republic of China, immunocompromised patients with malignant tumors are at high risk of severe illness and death following COVID-19 infection. Currently, there are no available data regarding the safety and efficacy of COVID-19 vaccines in cancer patients infected with severe acute respiratory syndrome coronavirus 2 (SARSCoV-2), the virus that causes COVID-19. The immune response and protective effects of vaccination may be reduced in this population. For inactivated vaccines and recombinant subunit vaccines, vaccination is recommended based on the safety profiles of previous vaccines of the same type. Although the viral vectors for adenoviral vector vaccines used are replication deficient, there are no existing safety data on the use of the same vaccine types. Therefore, 
cancer patients should be fully informed about the potential benefits and risks of COVID-19 vaccination.

The National Comprehensive Cancer Network COVID-19 Vaccination Advisory Committee strongly recommends that patients with cancer and those receiving anticancer therapy be prioritized for COVID-19 vaccination and that only authorized COVID-19 vaccines or approved for use by the United States Food and Drug Administration should be administered (9). However, people with special tumor-related conditions and those who recently developed COVID-19 may choose to delay vaccination. For patients receiving hematopoietic stem cell transplantation or cytokine therapy, COVID-19 vaccination should be delayed for 3 months.

During the COVID-19 pandemic, the diagnosis and treatment patterns of breast cancer have changed according to the specific grade and stage of malignancy $(10,11)$ to ensure patient safety. However, there is no direct evidence of whether patients with breast cancer can receive COVID-19 vaccination and on the timing of vaccination. The COVID-19 vaccination rate remains low among breast cancer patients in China, which can be explained by the inadequate health education and lack of vaccine safety data rather than vaccine shortage. Second, only small COVID-19 outbreaks occur in China, and many cancer patients think that COVID-19 vaccines are useless or may even increase the risk of tumor recurrence. The lack of data on the effectiveness of COVID-19 vaccine causes hesitancy among patients with breast cancer.

To promote the rational COVID-19 vaccination in breast cancer patients (12), the Chinese Society of Clinical Oncology-Breast Cancer held an online expert meeting on September 9, 2021, during which participants voted on issues related to vaccination for breast cancer patients in China and created expert recommendations on different breast cancer grades to guide clinical practice (Table 2).

\section{Recommendation grades}

* Grade I recommendation: strong consensus (>80\% agreement);

* Grade II recommendation: basic consensus (60-80\% agreement);

* Grade III recommendation: weak consensus $(<60 \%$ agreement).

\section{Determining whether breast cancer patients should receive COVID-19 vaccination when the condition allows}

Breast cancer patients are a special population, and few studies have investigated the safety and efficacy of COVID-19 vaccines in this population. Unfortunately, most breast cancer patients do not develop immunity against SARS-CoV-2 and are at an increased risk of severe illness and even death after being infected. Considering the clinical manifestations of patients with malignant tumors after inoculation with other vaccines, as well as the safety, efficacy, and necessity of COVID-19 vaccines, experts generally believe that breast cancer patients should receive the COVID-19 vaccine (Grade I recommendations).

\section{COVID-19 vaccination in patients with pathologically confirmed breast cancer and those scheduled for surgery}

Most experts suggest that vaccination should be determined on a case-by-case basis (13). Based on the global data on the overall safety profiles of COVID-19 vaccines, surgery may be performed after a short observation period following the vaccination in patients who have not yet received the vaccines to achieve timely treatment of breast cancer while fighting against COVID-19. If the possible side effects of the vaccination will affect the surgical processes and delay wound healing, surgery may be performed first, followed by COVID-19 vaccination at proper time points according to the patient's postoperative recovery status (Grade II recommendations).

For patients who received the first dose of COVID-19 vaccine but did not obtain the second dose, if a surgery is scheduled shortly, they may undergo surgery 1 week after the first dose or 1 week before the second dose; otherwise, the surgery should be postponed (Grade I recommendations).

\section{Optimal interval for surgery in breast cancer patients who have completed COVID-19 vaccination}

For patients who have completed the COVID-19 vaccination, surgery should be performed at least 1 week after vaccination, allowing adequate assessment of vaccinerelated reactions and not delaying the surgery (Grade I 
Table 2 Results of expert voting on consensuses and consensus grading

\begin{tabular}{|c|c|c|c|}
\hline Question & Yes & No & Abstain \\
\hline $\begin{array}{l}\text { Can patients who have not yet received the vaccine but are planning to have surgery in the } \\
\text { near future receive the COVID-19 vaccine? }\end{array}$ & $33 \%$ & $42 \%$ & $25 \%$ \\
\hline Should surgery be at least 1 week apart in patients who have already been vaccinated? & $100 \%$ & $0 \%$ & $0 \%$ \\
\hline $\begin{array}{l}\text { Are breast cancer patients one week before surgery to one month after surgery feasible for } \\
\text { COVID-19 vaccination? }\end{array}$ & $51 \%$ & $49 \%$ & $9 \%$ \\
\hline $\begin{array}{l}\text { Is COVID-19 vaccination feasible for breast cancer patients who are currently receiving } \\
\text { neoadjuvant chemotherapy? }\end{array}$ & $15 \%$ & $76 \%$ & $9 \%$ \\
\hline $\begin{array}{l}\text { Is COVID-19 vaccination feasible for breast cancer patients who are scheduled to receive } \\
\text { adjuvant chemotherapy? }\end{array}$ & $32 \%$ & $66 \%$ & $2 \%$ \\
\hline Can COVID-19 vaccination be initiated within 1 month after the last chemotherapy session? & $11 \%$ & $89 \%$ & $0 \%$ \\
\hline $\begin{array}{l}\text { Can COVID-19 vaccination be initiated within 1-3 month after the last chemotherapy } \\
\text { session? }\end{array}$ & $82 \%$ & $16 \%$ & $2 \%$ \\
\hline Can COVID-19 vaccination be initiated 3-month after the last chemotherapy session? & $85 \%$ & $4 \%$ & $11 \%$ \\
\hline $\begin{array}{l}\text { Is COVID-19 vaccination feasible for breast cancer patients who are currently receiving } \\
\text { adjuvant targeted therapy? }\end{array}$ & $80 \%$ & $11 \%$ & $9 \%$ \\
\hline Is COVID-19 vaccination feasible during oral capecitabine treatment? & $24 \%$ & $73 \%$ & $2 \%$ \\
\hline Is COVID-19 vaccination feasible during oral pyrotinib treatment? & $51 \%$ & $49 \%$ & $0 \%$ \\
\hline Is COVID-19 vaccination feasible during treatment with CDK4/ 6 inhibitors? & $24 \%$ & $76 \%$ & $0 \%$ \\
\hline Is COVID-19 vaccination feasible during treatment with HDAC inhibitors? & $4 \%$ & $91 \%$ & $5 \%$ \\
\hline Is COVID-19 vaccination feasible during treatment with PD1/PDL1 inhibitors? & $0 \%$ & $97 \%$ & $3 \%$ \\
\hline Are all patients in the new drug clinical trials eligible for COVID-19 vaccination? & $9 \%$ & $85 \%$ & $7 \%$ \\
\hline $\begin{array}{l}\text { Do you agree that the decision to receive COVID-19 vaccines for patients participating in } \\
\text { clinical trials should be made according to the specific study drug and protocol? }\end{array}$ & $66 \%$ & $23 \%$ & $11 \%$ \\
\hline Can patients receive the COVID-19 vaccines if they are in the subject screening period? & $58 \%$ & $40 \%$ & $2 \%$ \\
\hline $\begin{array}{l}\text { Can the COVID-19 vaccines be administered during the initial phase of the study } \\
\text { (first } 6 \text { months)? }\end{array}$ & $18 \%$ & $78 \%$ & $4 \%$ \\
\hline Can the COVID-19 vaccines be administered during the maintenance phase of the study? & $91 \%$ & $7 \%$ & $2 \%$ \\
\hline
\end{tabular}


recommendations).

\section{Surgical modalities and COVID-19 vaccination in breast cancer patients}

Different surgical procedures can result in variations in wound areas/degrees and recovery speeds, and vaccination may affect the postoperative recovery status. Some experts believe that there is no positive correlation between surgical extent and wound degree. Therefore, the decision to receive the COVID-19 vaccine was not strongly associated with the surgical modalities (Grade III recommendations).

Breast cancer patients in the perioperative period (1 week before surgery to 1 month after surgery) are should not receive the COVID-19 vaccine as it may have an effect on the patient's physical recovery and healing of surgical wounds (Grade II recommendations).

\section{Timing for COVID-19 vaccination among breast cancer patients receiving neoadjuvant treatment}

Experts recommend that the feasibility and timing of COVID-19 vaccination should be considered according to the neoadjuvant treatments used.

Chemotherapy weakens the immune system in patients receiving neoadjuvant chemotherapy. As COVID-19 is well under control in China, and neoadjuvant chemotherapy typically lasts for a relatively short period, COVID-19 vaccination may be postponed in these patients if they are under strict safety management (Grade II recommendations).

Neoadjuvant endocrine therapy causes fewer side effects. In addition, most patients in this subpopulation are relatively old, and the course of neoadjuvant endocrine therapy is long. Thus, these patients are at a higher risk of developing COVID-19. Therefore, experts recommend that patients undergoing preoperative neoadjuvant endocrine therapy receive COVID-19 vaccination after undergoing proper evaluation (Grade II recommendations).

\section{COVID-19 vaccination in breast cancer patients undergoing adjuvant chemotherapy}

Immune function is affected during adjuvant chemotherapy, which may increase the side effects of COVID-19 vaccines. Thus, the COVID-19 vaccination must be suspended in patients with breast cancer during the course of adjuvant chemotherapy (Grade I recommendations).
If a scheduled postoperative adjuvant chemotherapy for breast cancer has not yet started, COVID-19 vaccination should be initiated after the completion of adjuvant chemotherapy. The vaccination can be completed as planned for patients who have already received their first dose of the COVID-19 vaccine. However, concurrent chemotherapy should be avoided (Grade II recommendations).

\section{Interval for COVID-19 vaccination after chemotherapy}

Chemotherapy has a significant impact on a patient's physical condition. Hematopoietic and immune functions may not recover within 1 month after the last postoperative adjuvant chemotherapy, and recombinant human granulocyte-colony stimulating factor may be used to promote hematopoiesis. Thus, COVID-19 vaccination during this period was not recommended (Grade I recommendations).

COVID-19 vaccination should be initiated 3 months after the last chemotherapy session (Grade I recommendation). Physical recovery can be good 13 months after the last chemotherapy session, and COVID-19 vaccination during this period may be acceptable (Grade II recommendations).

\section{COVID-19 vaccination during the course of targeted adjuvant therapy in breast cancer patients}

Postoperative adjuvant treatment with targeted therapy alone has milder side effects, but a long treatment duration (1 year). Patients are at a long-term risk of developing COVID-19. Hence, COVID-19 vaccination is recommended for these patients (Grade I recommendation). However, some experts argue that tumor-targeted therapy is associated with antigen-antibody binding, and the vaccines themselves may affect this immune response. Therefore, vaccine safety must be carefully monitored during vaccination.

\section{COVID-19 vaccination in patients receiving adjuvant endocrine therapy}

Postoperative adjuvant endocrine therapy generally uses tamoxifen and aromatase inhibitors with good safety profiles. In addition, adjuvant endocrine therapy can last up to $5-10$ years. COVID-19 vaccines should be administered 
after chemotherapy and adjuvant endocrine therapy (Grade I recommendation).

\section{COVID-19 vaccination during adjuvant radiotherapy}

Adjuvant radiotherapy usually takes longer after breast cancer surgery (about 1 month); exposure to radiation, the abscopal effect, and the impact on the hematological system during radiotherapy should also be considered. Therefore, COVID-19 vaccination during radiotherapy is not recommended; it can only be initiated after the completion of radiotherapy (Grade II recommendations).

\section{Criteria for vaccinating metastatic breast cancer patients and early-stage breast cancer patients}

The physical condition and immune function of patients with metastatic breast cancer (MBC) are quite different from those in early-stage patients, and the drug therapies administered are more complicated. Thus, the decision to vaccinate should be based on the criteria different from those for early-stage breast cancer patients (Grade II recommendations).

\section{COVID-19 vaccination during oral capecitabine treatment}

COVID-19 vaccination can be considered in patients receiving early adjuvant treatment with oral capecitabine and in those who have good tolerance to this treatment (Grade I recommendation). COVID-19 vaccination can also be considered in $\mathrm{MBC}$ patients receiving maintenance capecitabine therapy and in those with stable disease who are responsive to this treatment (Grade II recommendation). For MBC patients with advanced-stage cancer, the decision to administer the COVID-19 vaccine or not should be made cautiously (Grade III recommendations).

\section{COVID-19 vaccination in patients taking oral pyrotinib, CDK4/6 inhibitors, and chidamide}

The mechanisms of action of new antitumor drugs, such as pyrotinib, CDK4/6 inhibitors, and chidamide, and their potential interactions with COVID-19 vaccines remain unclear. Thus, COVID-19 vaccination is, in principle, not recommended for patients treated with these drugs (Grade II recommendations).

\section{COVID-19 vaccination during treatment with PD1/PDL1 inhibitors}

The use of PD1/PDL1 inhibitors may affect the immune function; therefore, COVID-19 vaccination is not recommended in patients using PD1/PDL1 inhibitors (Grade I recommendations).

\section{Feasibility of COVID-19 vaccination during clinical trials of new drugs}

All foreign guidelines recommend COVID-19 vaccination for patients participating in clinical trials (14). However, because COVID-19 is well under control in China, and vaccination may affect the interpretation of the results of clinical trials, not all patients participating in clinical trials are suitable to receive the COVID-19 vaccine (Grade I recommendation). Hence, a decision on COVID-19 vaccination should be made based on the specific protocols of clinical trials (Grade II recommendations).

Experts believe that vaccinating patients during the participant screening period should be made with caution (Grade II recommendation). However, if a patient has already received the first dose of the COVID-19 vaccine before the participant screening period, he or she can complete the vaccination as planned, and then the suitability or eligibility for the study will be re-considered. If the patient has already entered the participant screening period, the COVID-19 vaccination must be suspended, as vaccination may affect the patient's safety assessment (Grade II recommendations).

COVID-19 vaccination should be avoided in patients during the initial phases of clinical trials (e.g., the first 6 months of salvage treatment for advanced breast cancer) to minimize the potential impact of vaccination on clinical interventions (Grade II recommendation). Patients who developed a stable disease after treatment and entered the study maintenance phase may receive the COVID-19 vaccine (Grade II recommendation). For patients participating in clinical studies on early neoadjuvant therapy or adjuvant therapy, a decision on COVID-19 vaccination should be made based on the specific treatment protocol, referring to the above expert opinion, or by a discussion with the principal investigator (PI) and/or sponsor.

There is a need for the administration of COVID-19 vaccines in patients with breast cancer. However, due to the lack of medical evidence on the effectiveness and safety of COVID-19 vaccines, the above expert opinions will 
serve as reference only. Before administering COVID-19 vaccines in breast cancer patients, the stability of the disease, the safety of the current treatments, and the expected survival of patients with advanced disease should be considered. Written informed consent must be obtained. Patients are advised to consult their healthcare providers on whether they are suited for COVID-19 vaccination; they may even refuse vaccination accordingly. The safety of COVID-19 vaccination in breast cancer patients should be adequately assessed, and COVID-19 vaccines should be administered to these patients in a timely and reasonable manner to ensure the safety of patients with cancer and COVID-19.

\section{Members of the expert panel}

Junwen Bai (Affiliated Hospital of Inner Mongolia Medical University), Bo Chen (First Affiliated Hospital of China Medical University), Yiding Chen (Second Affiliated Hospital of Medical College of Zhejiang University), Zhanhong Chen (Cancer Hospital Affiliated to University of Chinese Academy of Sciences), Peifen Fu (First Affiliated Hospital of Medical College of Zhejiang University), Rui Ge (Fudan University Affiliated Huadong Hospital), Cuizhi Geng (Fourth Hospital Affiliated to Hebei Medical University), Chunfang Hao (Tumor Hospital of Tianjin Medical University), Bin Hua (Beijing Hospital), Xuening Ji (Zhongshan Hospital Affiliated to Dalian University), Zefei Jiang (Fifth Medical Center of Chinese PLA General Hospital), Jianbin Li (Fifth Medical Center of Chinese PLA General Hospital), Man Li (Second Affiliated Hospital of Dalian Medical University), Ying Lin (First Affiliated Hospital of Sun Yat-sen University), Jian Liu (Fujian Cancer Hospital), Shu Liu (Guizhou Maternal and Child Health Hospital), Jie Ma (Tangshan People's Hospital of Hebei Province), Xueli Mo (Peking University Shougang Hospital), Jianyun Nie (Yunnan Cancer Hospital), Wenxing Qin (Shanghai Changzheng Hospital), Jing Shi (First Affiliated Hospital of China Medical University), Chuangui Song (Union Medical College Hospital Affiliated to Fujian Medical University), Yuhua Song (Affiliated Hospital of Qingdao University), Tao Sun (Liaoning Cancer Hospital), Zhengkui Sun (Jiangxi Cancer Hospital), Yue'e Teng (First Affiliated Hospital of China Medical University), Biyun Wang (Cancer Hospital Affiliated to Fudan University), Tao Wang (Fifth Medical Center of PLA General Hospital), Xiaojia Wang (Cancer Hospital Affiliated to Chinese Academy of Sciences), Ling Xu (Peking University First
Hospital), Ying Yan (Cancer Hospital of Peking University), Hua Yang (Affiliated Hospital of Hebei University), Songqing Ye (Fujian Provincial Hospital),Yongmei Yin(the First affiliation hospital with Nanjing medical university), Peng Yuan (Cancer Hospital of Chinese Academy of Medical Sciences), Jun Zhang (Fourth Hospital Affiliated to Hebei Medical University), Shaohua Zhang (Fifth Medical Center of PLA General Hospital), and Yanxia Zhao (Wuhan Union Hospital).

\section{Acknowledgments}

Funding: This article is supported by Emergency Key Program of Guangzhou Laboratory, Grant No. EKPG21-30-4.

\section{Footnote}

Conflicts of Interest: All authors have completed the ICMJE uniform disclosure form (available at https://dx.doi. org/10.21037/tbcr-21-31). JL serves as an unpaid Managing Editor of Translational Breast Cancer Research. YY, XW, CG and ES serve as the unpaid editorial board members of Translational Breast Cancer Research. ZJ serves as an unpaid Editor-in-Chief of Translational Breast Cancer Research. The other authors have no conflicts of interest to declare.

Ethical Statement: The authors are accountable for all aspects of the work in ensuring that questions related to the accuracy or integrity of any part of the work are appropriately investigated and resolved.

Open Access Statement: This is an Open Access article distributed in accordance with the Creative Commons Attribution-NonCommercial-NoDerivs 4.0 International License (CC BY-NC-ND 4.0), which permits the noncommercial replication and distribution of the article with the strict proviso that no changes or edits are made and the original work is properly cited (including links to both the formal publication through the relevant DOI and the license). See: https://creativecommons.org/licenses/by-nc-nd/4.0/.

\section{References}

1. World Health Organization. WHO Coronavirus Disease (COVID-19) Dashboard. Available online: https://covid19. who.int/

2. Lin $\mathrm{Y}, \mathrm{Hu} \mathrm{Z}$, Zhao Q, et al. Understanding COVID-19 vaccine demand and hesitancy: A nationwide online survey 
in China. PLoS Negl Trop Dis 2020;14:e0008961.

3. National Health Commission of The People's Republic of China. Vaccination of COVID-19. Available online: http:// www.nhc.gov.cn/jkj/s7915/202109/47aad44bd9b54b59a33 d29f7f7c2ca9e.shtmL.

4. Siegel RL, Miller KD, Fuchs HE, et al. Cancer Statistics, 2021. CA Cancer J Clin 2021;71:7-33.

5. Wu Z, McGoogan JM. Characteristics of and Important Lessons From the Coronavirus Disease 2019 (COVID-19) Outbreak in China: Summary of a Report of 72314 Cases From the Chinese Center for Disease Control and Prevention. JAMA 2020;323:1239-42.

6. Pathania AS, Prathipati P, Abdul BA, et al. COVID-19 and Cancer Comorbidity: Therapeutic Opportunities and Challenges. Theranostics 2021;11:731-53.

7. Guidelines Working Committee of Chinese Society of Clinical Oncology. Guidelines of Chinese Society of Clinical Oncology (CSCO) Breast Cancer 2021. Beijing: People's Medical Publishing House, 2021.

8. National Health Commission of the People's Republic of China. Technical Guide to COVID-19 Vaccination (first edition). Chin J Clin Infect Dis 2021;14:89-90.

doi: $10.21037 /$ tbcr-21-31

Cite this article as: Nie J, Wang T, Xu R, Li J, Yin Y, Wang X, Geng C, Wang FS, Song E, Jiang Z. Chinese expert consensus on coronavirus disease 2019 vaccination for breast cancer patients. Transl Breast Cancer Res 2021;2:34.
9. NCCN. Cancer and COVID-19 vaccination, 2021, version 4.0. Available online: https://wwwnccnorg/docs/defaultsource/covid-19/2021_covid-19_vaccination_guidance_v40pdf?sfvrsn=b483da2b_70

10. Li J, Wang H, Geng C, et al. Suboptimal declines and delays in early breast cancer treatment after COVID-19 quarantine restrictions in China: A national survey of 8397 patients in the first quarter of 2020. EClinicalMedicine 2020;26:100503.

11. Li F, Xu F, Zhang H, et al. Analysis of the treatment patterns and safety of early breast cancer patients during the COVID-19 pandemic. Transl Breast Cancer Res 2020;1:15.

12. Laine C, Cotton D, Moyer DV. COVID-19 Vaccine: Promoting Vaccine Acceptance. Ann Intern Med 2021;174:252-3.

13. Ko G, Hota S, Cil TD. COVID-19 vaccination and breast cancer surgery timing. Breast Cancer Res Treat 2021;188:825-6.

14. Desai A, Gainor JF, Hegde A, et al. COVID-19 vaccine guidance for patients with cancer participating in oncology clinical trials. Nat Rev Clin Oncol 2021;18:313-9. 